\title{
TRANSFORMATION FORMULAS FOR MULTIVARIABLE BASIC HYPERGEOMETRIC SERIES
}

\author{
T. H. Baker and P. J. Forrester
}

Dedicated to Dick Askey on the occasion of his 65th birthday

\begin{abstract}
We study multivariable (bilateral) basic hypergeometric series associated with (type $A$ ) Macdonald polynomials. We derive several transformation and summation properties for such series, including analogues of Heine's ${ }_{2} \phi_{1}$ transformation, the $q$-Pfaff-Kummer and Euler transformations, the $q$-Saalschütz summation formula, and Sear's transformation for terminating, balanced ${ }_{4} \phi_{3}$ series. For bilateral series, we rederive Kaneko's analogue of the ${ }_{1} \psi_{1}$ summation formula, and give multivariable extensions of Bailey's ${ }_{2} \psi_{2}$ transformations.
\end{abstract}

\section{Introduction}

Multivariable basic hypergeometric series of the type studied in this paper were first introduced by Kaneko [16] and Macdonald [19]. They are defined as

$$
{ }_{r} \Phi_{s}\left[\begin{array}{l}
a_{1}, \ldots, a_{r} \\
b_{1}, \ldots, b_{s}
\end{array} ;\right]:=\sum_{\lambda}\left((-1)^{|\lambda|} q^{n\left(\lambda^{\prime}\right)}\right)^{s+1-r} \frac{\left(a_{1} ; q\right)_{\lambda} \cdots\left(a_{r} ; q\right)_{\lambda}}{\left(b_{1} ; q\right)_{\lambda} \cdots\left(b_{s} ; q\right)_{\lambda} h_{\lambda}^{\prime}} P_{\lambda}(z ; q, t)
$$

where all quantities are defined in Section 1.1.

The $q=1$ case had been studied previously by Yan [24] where several important properties of the hypergeometric series ${ }_{p} \mathcal{F}_{q}$ were studied, including the Gauss $\left({ }_{2} \mathcal{F}_{1}\right)$ and Kummer $\left({ }_{1} \mathcal{F}_{1}\right)$ summation formulas, and the Pfaff-Kummer and Euler transformations, and integral representations were derived (see also [22] for the Gauss formula with respect to arbitrary root systems).

Kaneko [16] considered a generalized $q$-Selberg integral dependent on parameters $x_{1}, \ldots, x_{m}$ and derived a set of $m q$-difference equations satisfied by such an integral. He then showed that the basic hypergeometric series ${ }_{2} \Phi_{1}$ defined by (1.1) was the unique solution (satisfying certain properties) of such a system. Thus he was able to derive an integral representation for this ${ }_{2} \Phi_{1}$ series and hence give an alternative proof of the $q$-Selberg integral [2] (see also [11, 12, 25]). He additionally derived the $q$-analogue of the Gauss formula and another integral formula, the constant term version of which was presented in [18, Theorem 4]. In a subsequent work [14], Kaneko introduced a multivariable analogue of the bilateral basic hypergeometric series $r \Psi_{s+1}$ and derived an analogue of Ramanujan's ${ }_{1} \psi_{1}$ summation formula, along with a multivariable version of the Jacobi triple product identity (which is a limiting case; see also [17]).

Independently, Macdonald, in his unpublished notes [19], carried out (among other things) a program similar to Yan in the $q=1$ case along with a multivariable version

Received March 27, 1998, revised August 14, 1998.

1991 Mathematics Subject Classification: 33D80. 
of the Saalschütz summation formula (the summation of a balanced ${ }_{3} \mathcal{F}_{2}$ with unit argument), while for general $q$, he derived the integral representation for the ${ }_{2} \Phi_{1}$ series and $q$-analogues of the Gauss and Saalschütz formulas.

The aim of the present work is to supplement some of the existing knowledge with some new transformation and summation formulas in this multivariable setting (including the $q$-analogue of the Pfaff-Kummer transformation formulas, Heine's transformation formula for the ${ }_{2} \Phi_{1}$ series, Sear's transformation for terminating, balanced ${ }_{4} \Phi_{3}$ series, and various summation and transformation formulas for bilateral ${ }_{2} \Psi_{2}$ series), as well as providing alternative derivations of known results. For a comprehensive review of summation and transformation formulas for basic hypergeometric series in the one-variable case, see Gasper and Rahman's book [7].

We note here that many of the summation and transformation formulas involving multivariable basic hypergeometric series are only valid when the argument is specialized to $z t^{\delta}:=\left(z, z t, z t^{2}, \ldots, z t^{n-1}\right)$ (the exceptions being the $q$-binomial theorem, the Euler transformation of the ${ }_{2} \Phi_{1}$ series, and the ${ }_{1} \Psi_{1}$ summation formula).

Finally, we point out that many of the formulas presented here can be derived in the case $q=t$ (the Schur polynomial case) as special cases of the very general formulas of Milne and co-workers (see [21] and references therein), or from those found in the works of Krattenthaler, Gustafson, and Schlosser [8, 23]. Also, summation formulas for hypergeometric systems associated with the $B C$ root system have been considered by Van Diejen [4] (see also [5]).

The plan of the paper is as follows. In Section 1.1, we set out the basic facts about Macdonald polynomials that we shall require. Section 2 exhibits a multivariable extension of Heine's ${ }_{2} \phi_{1}$ transformations. The Euler transformation is derived in Section 3 from the defining difference equations for the ${ }_{2} \Phi_{1}$ series. Section 4 treats the $q$-Saalschütz formula, while the $q$-Pfaff-Kummer transformation for ${ }_{2} \Phi_{1}$ and Sear's transformation for terminating, balanced ${ }_{4} \Phi_{3}$ series are discussed in Section 5 . Bilateral series are studied in Section 6 where the ${ }_{1} \Psi_{1}$ summation formula is derived using a shifted version of the multivariable Gauss summation formula, and various ${ }_{2} \Psi_{2}$ transformations are derived using two different methods.

1.1. Notations. The Macdonald polynomials $P_{\lambda}(x ; q, t), x:=\left(x_{1}, \ldots, x_{n}\right)$ (often abbreviated as $P_{\lambda}(x)$ or just $P_{\lambda}$ when the context is clear) are defined as the unique symmetric polynomials having the expansion

$$
P_{\lambda}(x)=m_{\lambda}(x)+\sum_{\mu<\lambda} c_{\lambda \mu} m_{\mu}(x)
$$

(where $<$ denotes the dominance order on partitions and $m_{\lambda}(x)$ denotes the monomial symmetric function), which form an orthogonal basis of symmetric functions with respect to the inner product

$$
\langle f, g\rangle_{q, t}:=\frac{1}{n !} \text { C.T. }\left(f(x) g\left(x^{-1}\right) \Delta_{q, t}(x)\right)
$$

where C.T. stands for "the constant term of" in the case of Laurent polynomials or the corresponding contour integral otherwise, $|q|<1,(u ; q)_{\infty}:=\prod_{i=0}^{\infty}\left(1-u q^{i}\right)$, $x^{-1}:=\left(x_{1}^{-1}, \ldots, x_{n}^{-1}\right)$, and

$$
\Delta_{q, t}(x):=\prod_{1 \leq i<j \leq n} \frac{\left(x_{i} / x_{j} ; q\right)_{\infty}\left(x_{j} / x_{i} ; q\right)_{\infty}}{\left(t x_{i} / x_{j} ; q\right)_{\infty}\left(t x_{j} / x_{i} ; q\right)_{\infty}}
$$


We define the quantities

$$
h_{\lambda}=\prod_{s \in \lambda}\left(1-q^{a(s)} t^{l(s)+1}\right), \quad h_{\lambda}^{\prime}=\prod_{s \in \lambda}\left(1-q^{a(s)+1} t^{l(s)}\right)
$$

where $a(s)=\lambda_{i}-j$ and $l(s)=\lambda_{j}^{\prime}-i$ denote the arm-length and leg-length (that is, the number of boxes to the right of and below, but not including $s$ ), respectively, of the node $s=(i, j)$ in the Ferrer's diagram of $\lambda$. The generalized shifted $q$-factorial is given by $(a ; q)_{\lambda}:=t^{n(\lambda)} \prod_{i=1}^{n}\left(a t^{1-i} ; q\right)_{\lambda_{i}}$. Here $n(\lambda)=\sum_{i}(i-1) \lambda_{i}=\sum_{j} \lambda_{j}^{\prime}\left(\lambda_{j}^{\prime}-1\right) / 2$. We require two fundamental results on Macdonald polynomials, both of which can be found in [20]. They are the specialization formula

$$
P_{\lambda}\left(1, t, \ldots, t^{n-1}\right)=\frac{\left(t^{n} ; q\right)_{\lambda}}{h_{\lambda}}
$$

and the normalization with respect to the inner product (2.1)

$$
\left\langle P_{\lambda}, P_{\lambda}\right\rangle_{q, t}=\frac{h_{\lambda}^{\prime}}{h_{\lambda}} \frac{\left(t^{n} ; q\right)_{\lambda}}{\left(q t^{n-1} ; q\right)_{\lambda}}\langle 1,1\rangle_{q, t} .
$$

In the case $t=q^{k}$ where $k$ is a positive integer, we have the explicit evaluation $[9,11,25]$

$$
\langle 1,1\rangle_{q, q^{k}}=\frac{1}{n !} \text { C.T. } \Delta_{k}(x)=\prod_{i=1}^{n} \frac{(q ; q)_{i k-1}}{(q ; q)_{k-1}(q ; q)_{(i-1) k}} .
$$

We note that in general

$$
\langle 1,1\rangle_{q, t}=\frac{1}{n !} \text { C.T. } \Delta_{q, t}(x)=\prod_{i=1}^{n} \frac{(t ; q)_{\infty}\left(q t^{i-1} ; q\right)_{\infty}}{\left(t^{i} ; q\right)_{\infty}(q ; q)_{\infty}}
$$

where the product $\Delta_{q, t}(x)$ is expanded using the $q$-binomial theorem

$$
\sum_{n \geq 0} \frac{(a ; q)_{n}}{(q ; q)_{n}} z^{n}=\frac{(a z ; q)_{\infty}}{(z ; q)_{\infty}}
$$

The identity (1.8) can be proved by applying Ismail's argument as found in [10] using the known result (1.7). Similarly, although the result (1.6) is derived in ref. [20] with the restriction $t=q^{k}$, the final result remains valid for general $t$ (see the remark on p. 372 of $[20])$.

Further, following Macdonald [20], for a partition $\lambda$, let $u_{\lambda}$ denote the evaluation map on polynomials in $n$ variables which sets $x_{i}=q^{\lambda_{i}} t^{n-i}$. There are no nice expressions for $u_{\lambda}\left(P_{\mu}\right)$ except in the cases $\lambda=0$ (since $u_{0}\left(P_{\mu}\right)=P_{\mu}\left(1, t, \ldots, t^{n-1}\right.$ ) given above in (1.5) or more generally $\lambda=\left(m^{n}\right)$ (since $u_{\left(m^{n}\right)}\left(P_{\mu}\right)=q^{m} u_{0}\left(P_{\mu}\right)$ ). Nonetheless, there is a useful symmetry property for $u_{\lambda}\left(P_{\mu}\right)$ in general, which reads

$$
\frac{u_{\lambda}\left(P_{\mu}\right)}{u_{0}\left(P_{\mu}\right)}=\frac{u_{\mu}\left(P_{\lambda}\right)}{u_{0}\left(P_{\lambda}\right)} \text {. }
$$

One of the fundamental results in the theory of basic hypergeometric series is the summation formula for $\mathrm{a}_{1} \phi_{0}$ series (1.9). This has the important multivariable generalization (see e.g., [16, Thm 3.5]):

$$
{ }_{1} \Phi_{0}[\stackrel{a}{-} ; z]=\prod_{i=1}^{n} \frac{\left(a z_{i} ; q\right)_{\infty}}{\left(z_{i} ; q\right)_{\infty}}
$$


which will often be of use below.

\section{Heine's transformation formula}

A fundamental transformation formula for ${ }_{2} \phi_{1}$ series is Heine's transformation formula

$$
{ }_{2} \phi_{1}\left[\begin{array}{c}
a, b \\
c
\end{array} ; z\right]=\frac{(b ; q)_{\infty}(a z ; q)_{\infty}}{(c ; q)_{\infty}(z ; q)_{\infty}}{ }_{2} \phi_{1}\left[\begin{array}{c}
c / b, z \\
a z
\end{array} ; b\right] .
$$

The utility of this formula is two-fold: iterate it twice to get the Euler transformation formula (see (3.1)); set $z=c / a b$ and the ${ }_{2} \phi_{1}$ on the right-hand side of (2.1), becomes ${ }_{1} \phi_{0}$ which can be summed by the $q$-binomial theorem resulting in the Gauss summation formula

$$
{ }_{2} \phi_{1}\left[\begin{array}{c}
a, b \\
c
\end{array} ; \frac{c}{a b}\right]=\frac{(c / a ; q)_{\infty}(c / b ; q)_{\infty}}{(c ; q)_{\infty}(c / a b ; q)_{\infty}} .
$$

The multivariable version of Heine's formula is:

$$
{ }_{2} \Phi_{1}\left[\begin{array}{c}
a, b \\
c
\end{array} ; z t^{\delta}\right]=\prod_{i=1}^{n} \frac{\left(b t^{1-i} ; q\right)_{\infty}\left(a z t^{n-i} ; q\right)_{\infty}}{\left(c t^{1-i} ; q\right)_{\infty}\left(z t^{n-i} ; q\right)_{\infty}} \Phi_{1}\left[\begin{array}{c}
c / b, z t^{n-1} \\
a z t^{n-1} ; b t^{1-n} t^{\delta}
\end{array}\right]
$$

(as mentioned in the introduction, the notation $z t^{\delta}$ stands for the argument $(z, z t, \ldots$, $\left.z t^{n-1}\right)$ ). The proof of this identity follows in the same manner as the one-variable case, with the aid of the symmetry property (1.10). To see this, first note that setting $z_{i}=b t^{1-i} q^{\lambda_{i}}$ and $a=c / b$ in the $q$-binomial theorem (1.11) yields

$$
\prod_{i} \frac{\left(c t^{1-i} q^{\lambda_{i}} ; q\right)_{\infty}}{\left(b t^{1-i} q^{\lambda_{i}} ; q\right)_{\infty}}=\sum_{\mu} \frac{(c / b ; q)_{\mu}}{h_{\mu}^{\prime}}\left(b t^{1-n}\right)^{|\mu|} u_{\lambda}\left(P_{\mu}\right)
$$

Thus

$$
\begin{aligned}
{ }_{2} \Phi_{1}\left[\begin{array}{c}
a, b \\
c
\end{array} z t^{\delta}\right] & =\prod_{i=1}^{n} \frac{\left(b t^{1-i} ; q\right)_{\infty}}{\left(c t^{1-i} ; q\right)_{\infty}} \sum_{\lambda} \frac{(a ; q)_{\lambda}}{h_{\lambda}^{\prime}} P_{\lambda}\left(t^{\delta}\right) z^{|\lambda|} \sum_{\mu} \frac{(c / b ; q)_{\mu}}{h_{\mu}^{\prime}}\left(b t^{1-n}\right)^{|\mu|} u_{\lambda}\left(P_{\mu}\right) \\
& =\prod_{i=1}^{n} \frac{\left(b t^{1-i} ; q\right)_{\infty}}{\left(c t^{1-i} ; q\right)_{\infty}} \sum_{\lambda, \mu} \frac{(a ; q)_{\lambda}(c / b ; q)_{\mu}}{h_{\lambda}^{\prime} h_{\mu}^{\prime}} z^{|\lambda|}\left(b t^{1-n}\right)^{|\mu|} u_{0}\left(P_{\mu}\right) u_{\mu}\left(P_{\lambda}\right) \\
& =\prod_{i=1}^{n} \frac{\left(b t^{1-i} ; q\right)_{\infty}\left(a z t^{n-i} ; q\right)_{\infty}}{\left(c t^{1-i} ; q\right)_{\infty}\left(z t^{n-i} ; q\right)_{\infty}} \sum_{\mu} \frac{(c / b ; q)_{\mu}\left(z t^{n-1} ; q\right)_{\mu}}{\left(a z t^{n-1} ; q\right)_{\mu} h_{\mu}^{\prime}}\left(b t^{1-n}\right)^{|\mu|} u_{0}\left(P_{\mu}\right)
\end{aligned}
$$

which gives the result. Here we have used (2.3), (1.10), and (2.3), respectively.

As mentioned above, upon setting $z=c / a b t^{n-1}$, the right-hand side of (2.2) reduces to a ${ }_{1} \Phi_{0}$ which can be summed through the $q$-binomial theorem giving the Gauss formula

$$
{ }_{2} \Phi_{1}\left[\begin{array}{c}
a, b \\
c
\end{array} ; \frac{c}{a b t^{n-1}} t^{\delta}\right]=\prod_{i=1}^{n} \frac{\left(c t^{1-i} / b ; q\right)_{\infty}\left(c t^{1-i} / a ; q\right)_{\infty}}{\left(c t^{1-i} / a b ; q\right)_{\infty}\left(c t^{1-i} ; q\right)_{\infty}} .
$$

Also, we can iterate (2.2) twice and obtain a version of Euler's transformation which is valid for the variables $z t^{\delta}$. In fact, we shall see in the next section that there exists a multivariable version of the Euler transformation, which is true for general argument $z:=\left(z_{1}, \ldots, z_{n}\right)$. 


\section{Euler transformation}

In the theory of the one-variable $q$-hypergeometric function ${ }_{2} \phi_{1}$, the Euler transformation reads

$$
{ }_{2} \phi_{1}\left[\begin{array}{c}
a, b \\
c
\end{array} ; x\right]=\frac{(a b x / c ; q)_{\infty}}{(x ; q)_{\infty}}{ }_{2} \phi_{1}\left[\begin{array}{c}
c / a, c / b \\
c
\end{array} ; a b x / c\right] .
$$

This transformation generalizes naturally to the $n$-variable case.

Proposition 3.1. With ${ }_{2} \Phi_{1}$ defined by (1.1), we have

$$
{ }_{2} \Phi_{1}\left[\begin{array}{c}
a, b \\
c
\end{array} ; z\right]=\prod_{i=1}^{n} \frac{\left(a b z_{i} / c ; q\right)_{\infty}}{\left(z_{i} ; q\right)_{\infty}}{ }_{2} \Phi_{1}\left[\begin{array}{c}
c / a, c / b \\
c
\end{array} ; a b z / c\right] .
$$

The $n$-variable Euler transformation (3.2) can be proved by using a theorem of Kaneko [16, Thm. 4.12] which characterizes ${ }_{2} \Phi_{1}$ as the unique solution of a system of $q$-difference equations. To state these equations, let $\tau_{i}$ denote the $q$-shift operator for the variable $z_{i}$ so that $\tau_{i} f\left(z_{1}, \ldots, z_{n}\right)=f\left(z_{1}, \ldots, q z_{i}, \ldots, z_{n}\right)$, write

$$
A_{i}(z ; t):=\prod_{\substack{l=1 \\ l \neq i}}^{n} \frac{t z_{i}-z_{l}}{z_{i}-z_{l}}
$$

and define the $q$-derivative by

$$
\frac{\partial \phi}{\partial_{q} z_{i}}=\frac{\left(1-\tau_{i}\right) \phi}{(1-q) z_{i}}
$$

Theorem 3.2 (Kaneko). The multivariable basic q-hypergeometric series ${ }_{2} \Phi_{1}\left[\begin{array}{c}a, b \\ c\end{array} ; z\right]$ as defined by (1.1) is the unique solution of the system of $q$-difference equations ${ }^{1}$

$$
\begin{aligned}
z_{i}(c- & \left.a b q z_{i}\right) \tau_{i}\left(A_{i}(z ; t)\right) \frac{\partial^{2} S}{\partial_{q} z_{i}^{2}}+(1-t) \sum_{\substack{j=1 \\
j \neq i}}^{n} \frac{z_{i} z_{j}\left(c-a b z_{j}\right)}{q z_{i}-t z_{j}} \tau_{i}\left(A_{j}(z ; t)\right) \frac{\partial^{2} S}{\partial_{q} z_{i} \partial_{q} z_{j}} \\
& +\left\{\frac{t^{n-1}-c}{1-q}+\frac{1}{1-q}\left((1-a)(1-b) t^{n-1}-\left(t^{n-1}-a b q\right)\right) z_{i}\right\} \frac{\partial S}{\partial_{q} z_{i}} \\
& +\frac{1-t}{1-q}\left\{\frac{1-\tau_{i}\left(A_{i}(z ; t)\right)}{1-t}\left(c-a b q z_{i}\right) \frac{\partial S}{\partial_{q} z_{i}}-\sum_{\substack{j=1 \\
j \neq i}}^{n} \frac{z_{j}\left(c-a b z_{j}\right)}{q z_{i}-t z_{j}} \tau_{i}\left(A_{j}(z ; t)\right) \frac{\partial S}{\partial_{q} z_{j}}\right\} \\
& -\frac{(1-a)(1-b) t^{n-1}}{(1-q)^{2}} S=0, \quad i=1, \ldots, n,
\end{aligned}
$$

subject to the conditions that $S(z)$ is a symmetric function in $z_{1}, \ldots, z_{n}$ and $S(z)$ is analytic at the origin with $S(0)=1$.

In light of Theorem 3.2, the Euler transformation (3.2) can be proved by verifying that the substitution

$$
S=\prod_{i=1}^{n} \frac{\left(a b z_{i} / c ; q\right)_{\infty}}{\left(z_{i} ; q\right)_{\infty}} U
$$

\footnotetext{
${ }^{1}$ This is $(2.26)$ of $[16]$ with the factor $(1-q)$ in the second term corrected to read $(1-t)$.
} 
into the $q$-difference equation (3.4) implies that $U$ satisfies the same $q$-difference equation (3.4) with the replacements

$$
a \mapsto \frac{c}{a}, \quad b \mapsto \frac{c}{b}, \quad c \mapsto c, \quad z_{i} \mapsto \frac{a b z_{i}}{c}
$$

To perform this task, we will require the $q$-differentiation product rule

$$
\frac{\partial(\phi \psi)}{\partial_{q} z_{i}}=\frac{\partial \phi}{\partial_{q} z_{i}} \psi+\left(\tau_{i} \phi\right) \frac{\partial \psi}{\partial_{q} z_{i}}
$$

as well as the special summation formula [16, eqn.(2.11)]

$$
\sum_{\substack{j=1 \\ j \neq i}}^{n} \frac{z_{j} A_{j}(z ; t)}{t z_{j}-z_{i}}=\frac{A_{i}(z ; t)-t^{n-1}}{1-t}
$$

Proof of Proposition 3.1. Let us now give some details of the required calculation. With

$$
P_{i}:=\frac{\left(a b z_{i} / c ; q\right)_{\infty}}{\left(z_{i} ; q\right)_{\infty}}, \quad P_{i}^{\prime \prime}:=\frac{\left(a b q^{2} z_{i} / c ; q\right)_{\infty}}{\left(z_{i} ; q\right)_{\infty}}
$$

straightforward use of (3.3) and (3.6) shows that

$$
\begin{aligned}
\frac{\partial}{\partial_{q} z_{i}}\left(P_{i} U\right)= & \frac{1-a b / c}{1-q}\left(1-a b q z_{i} / c\right) P_{i}^{\prime \prime} U+\left(1-z_{i}\right)\left(1-a b q z_{i} / c\right) P_{i}^{\prime \prime} \frac{\partial U}{\partial_{q} z_{i}} \\
\frac{\partial^{2}}{\partial_{q} z_{i}^{2}}\left(P_{i} U\right)= & \frac{(1-a b / c)(1-a b q / c)}{(1-q)^{2}} P_{i}^{\prime \prime} U+\frac{(1-a b / c)\left(1-z_{i}\right)}{1-q} P_{i}^{\prime \prime} \frac{\partial U}{\partial_{q} z_{i}} \\
& +\frac{q}{1-q}(1-a b / c)\left(1-z_{i}\right) P_{i}^{\prime \prime} \frac{\partial U}{\partial_{q} z_{i}}+\left(1-z_{i}\right)\left(1-q z_{i}\right) P_{i}^{\prime \prime} \frac{\partial^{2} U}{\partial_{q} z_{i}^{2}}
\end{aligned}
$$

Double use of (3.8) allows us to write down a similar formula for

$$
\frac{\partial^{2}}{\partial_{q} z_{i} \partial_{q} z_{j}}\left(P_{i} P_{j} U\right)
$$

Next we use these formulas to rewrite the derivatives in (3.4) with $S=\left(\prod_{i=1}^{n} P_{i}\right) U$, and proceed to collect together terms so that the essential structure of (3.4) is maintained. Thus, for example, we must collect together all terms proportional to $U$, and show that, apart from a common factor (which is eventually canceled out), the 
coefficient of $S$ in (3.4), with the substitutions (3.5), results. These terms are

$$
\begin{aligned}
& \frac{\left(1-a b q z_{i} / c\right)}{(1-q)^{2}}\left(\prod_{\substack{l=1 \\
l \neq i}}^{n} P_{l}\right) P_{i}^{\prime \prime}\left[c z_{i} \tau_{i}\left(A_{i}(z ; t)\right)(1-a b / c)(1-a b q / c)\right. \\
& \quad+(1-t) c(1-a b / c)^{2} z_{i} \sum_{\substack{j=1 \\
j \neq i}}^{n} \frac{z_{j}}{q z_{i}-t z_{j}} \tau_{i}\left(A_{j}(z ; t)\right) \\
& \quad+(1-a b / c)\left\{t^{n-1}-c+\left((1-a)(1-b) t^{n-1}-\left(t^{n-1}-a b q\right)\right) z_{i}\right\} \\
& \quad+(1-t)(1-a b / c)\left\{\frac{1-\tau_{i}\left(A_{i}(z ; t)\right)}{1-t}\left(c-a b q z_{i}\right)-c \sum_{\substack{j=1 \\
j \neq i}}^{n} \frac{z_{j}}{q z_{i}-t z_{j}} \tau_{i}\left(A_{j}(z ; t)\right)\right\} \\
& \left.\quad-(1-a)(1-b) t^{n-1}\left(1-a b z_{i} / c\right)\right] .
\end{aligned}
$$

Noting that

$$
\sum_{\substack{j=1 \\ j \neq i}}^{n} \frac{z_{j}}{q z_{i}-t z_{j}} \tau_{i}\left(A_{j}(z ; t)\right)=\tau_{i}\left(\sum_{\substack{j=1 \\ j \neq i}}^{n} \frac{z_{j}}{z_{i}-t z_{j}} A_{j}(z ; t)\right)
$$

we see that the summation can be evaluated according to (3.7). The expression (3.10) then greatly simplifies, reducing down to

$$
\left(\left(1-a b q z_{i} / c\right)\left(1-z_{i}\right)\left(\prod_{\substack{l=1 \\ l \neq i}}^{n} P_{l}\right) P_{i}^{\prime \prime}\right)\left\{-\frac{(1-a / c)(1-b / c) t^{n-1}}{(1-q)^{2}}\right\}
$$

The factor in the curly brackets is precisely what is required to obtain (3.4) with the replacements (3.5).

Similar calculations to simplify the terms proportional to the $q$-derivatives show that the precise $q$-difference equation (3.4) results with $S=U$ and the replacements (3.5); the term in the parenthesis in (3.11) is a common factor which cancels out.

\section{4. q-Saalschütz summation formula}

A summation formula for basic hypergeometric series used in many contexts is the sum of a terminating, balanced ${ }_{3} \phi_{2}$ series ( $q$-Saalschütz formula)

$$
{ }_{3} \phi_{2}\left[\begin{array}{c}
q^{-N}, b, c \\
e, q^{1-N} b c / e ;^{q}
\end{array}\right]=\frac{(e / b ; q)_{N}(e / c ; q)_{N}}{(e ; q)_{N}(e / b c ; q)_{N}} .
$$

This formula follows directly by comparing coefficients of $z^{N}$ on both sides of the Euler transformation formula (4.1) (the products being expanded with the $q$-binomial theorem). This can be generalized to a multi-variable setting following the proof of Macdonald [19] in the $q=1$ case. First, we need some preliminary results.

Given a partition $\lambda$ of length $\ell(\lambda) \leq n$, let $\hat{\lambda}$ be the complement of $\lambda$ in the rectangle $\left(N^{n}\right)$. That is, $\hat{\lambda}_{i}=N-\lambda_{n+1-i}$. Then it follows from the various definitions that

$$
(a ; q)_{\hat{\lambda}}=t^{n(\lambda)} q^{n\left(\lambda^{\prime}\right)-(N-1)|\lambda|} \frac{(a ; q)_{\left(N^{n}\right)}}{(-a)^{|\lambda|}\left(t^{n-1} q^{1-N} / a ; q\right)_{\lambda}} .
$$


Next, let $f_{\mu \nu}^{\lambda}(q, t)$ denote the coefficient of $P_{\lambda}$ in the expansion of $P_{\mu} P_{\nu}$. The following result is due to Macdonald [19], but we include the proof for completeness.

Lemma 4.1. We have

$$
f_{\mu \nu}^{\left(N^{n}\right)}(q, t)=\frac{\left\langle P_{\mu}, P_{\mu}\right\rangle_{k}}{\langle 1,1\rangle_{k}} \delta_{\hat{\mu} \nu}=\frac{\left(t^{n} ; q\right)_{\mu} h_{\mu}^{\prime}}{\left(q t^{n-1} ; q\right)_{\mu} h_{\mu}} \delta_{\hat{\mu} \nu}
$$

Proof. Writing $|x|:=x_{1} \cdots x_{n}$, first note that

$$
P_{\hat{\lambda}}(x)=|x|^{N} P_{\lambda}\left(x^{-1}\right)
$$

which follows from the characterization of the Macdonald polynomials explained in Section 1.1 (clearly both sides of the above equation have leading-order term $m_{\hat{\lambda}}(x)$ and also $\left\langle|x|^{N} P_{\lambda}\left(x^{-1}\right),|x|^{N} P_{\mu}\left(x^{-1}\right)\right\rangle_{k}=\left\langle P_{\mu}, P_{\lambda}\right\rangle_{k}$, yielding the requisite orthogonality; see also [5] on this point). It thus follows from (4.3) that

$$
\begin{aligned}
\left\langle P_{\lambda}(x), P_{\mu}(x)\right\rangle_{k} & =\left\langle P_{\lambda}(x) P_{\mu}\left(x^{-1}\right), 1\right\rangle_{k}=\left\langle P_{\lambda}(x) P_{\hat{\mu}}(x),|x|^{N}\right\rangle_{k} \\
& =\sum_{\sigma} f_{\lambda \hat{\mu}}^{\sigma}(q, t)\left\langle P_{\sigma}(x), P_{\left(N^{n}\right)}(x)\right\rangle_{k}=f_{\lambda \hat{\mu}}^{\left(N^{n}\right)}(q, t)\langle 1,1\rangle_{k} .
\end{aligned}
$$

The result now follows from the orthogonality of Macdonald polynomials and the expression (2.5) for their normalization.

The last ingredient needed is the following expression for $h_{\lambda}^{\prime}$ due to Kaneko [17]:

$$
\left.h_{\lambda}^{\prime}=(q ; q)_{\infty}^{n} \prod_{i} \frac{1}{\left(q^{\lambda_{i}+1} t^{n-i} ; q\right)_{\infty}} \prod_{i<j} \frac{\left(q^{\lambda_{i}-\lambda_{j}+1} t^{j-i} ; q\right)_{\infty}}{\left(q^{\lambda_{i}-\lambda_{j}+1} t^{j-i-1}\right.} ; q\right)_{\infty},
$$

from which one obtains a relation we shall need presently, namely,

$$
\frac{h_{\hat{\lambda}}^{\prime}}{h_{\lambda}^{\prime}}=(-1)^{|\lambda|} t^{n(\lambda)-n\left(\left(N^{n}\right)\right)} q^{n\left(\lambda^{\prime}\right)-N|\lambda|} \frac{\left(q t^{n-1} ; q\right)_{\left(N^{n}\right)}}{\left(q^{-N} ; q\right)_{\lambda}\left(q t^{n-1} ; q\right)_{\lambda}} .
$$

Beginning with the Euler transformation formula (3.2), expand the products occurring there with the $q$-binomial theorem and compare coefficients of $P_{\lambda}(z)$ on both sides to obtain

$$
\frac{(a ; q)_{\lambda}(b ; q)_{\lambda}}{(c ; q)_{\lambda} h_{\lambda}^{\prime}}=\sum_{\mu, \nu} \frac{(c / a ; q)_{\mu}(c / b ; q)_{\mu}(a b / c ; q)_{\nu}}{(c ; q)_{\mu} h_{\mu}^{\prime} h_{\nu}^{\prime}}\left(\frac{a b}{c}\right)^{|\mu|} f_{\mu \nu}^{\lambda}(q, t) .
$$

The point here is that, in the case when $\lambda=\left(N^{n}\right)$, the sum on the right-hand side can be expressed as a balanced ${ }_{3} \Phi_{2}$ series (the series ${ }_{r} \Phi_{s}$ in (1.1) is said to be balanced if $a_{1} \cdots a_{r} q t^{n-1}=b_{1} \cdots b_{s}$ ). Indeed, after using the expression (4.2) for $f_{\mu \nu}^{\left(N^{n}\right)}$ and expressing all quantities labeled by $\hat{\mu}$ in terms of those of $\mu$ by means of (4.1) and (4.5), the resulting sum over $\mu$ can be simplified to yield

$$
\frac{(a ; q)_{\left(N^{n}\right)}(b ; q)_{\left(N^{n}\right)}}{(c ; q)_{\left(N^{n}\right)}(a b / c ; q)_{\left(N^{n}\right)}}={ }_{3} \Phi_{2}\left[\begin{array}{c}
q^{-N}, c / a, c / b \\
c, c t^{n-1} q^{1-N} / a b
\end{array} ; q t^{\delta}\right],
$$

which is a multivariable version of the $q$-Saalschütz formula.

Note that by taking the limit $a \rightarrow 0$ in (4.6) and using the fact that

$$
\lim _{x \rightarrow \infty} \frac{(x ; q)_{\mu}}{x^{|\mu|}}=(-1)^{|\mu|} q^{n\left(\mu^{\prime}\right)}
$$


we have (after shifting $b \mapsto c / b$ )

$$
t^{n(\lambda)} \frac{(c / b ; q)_{\lambda}}{(c ; q)_{\lambda} h_{\lambda}^{\prime}}=\sum_{\mu, \nu} t^{n(\nu)} q^{n\left(\mu^{\prime}\right)} \frac{(b ; q)_{\mu}}{(c ; q)_{\mu} h_{\mu}^{\prime} h_{\nu}^{\prime}}\left(-\frac{c}{b}\right)^{|\mu|} f_{\mu \nu}^{\lambda}(q, t),
$$

which is a generalization of the Chu-Vandermonde formula (especially in the case $\lambda=\left(N^{n}\right)$ when the right-hand side can be expressed as a terminating $\left.{ }_{2} \Phi_{1}\right)$ that we shall need subsequently.

\section{Other transformation formulae}

One of the simplest transformations where the simple $n=1$ proof must be rethought is the Pfaff-Kummer transformation

$$
{ }_{2} \phi_{1}\left[\begin{array}{c}
a, b \\
c
\end{array} ; z\right]=\frac{(a z ; q)_{\infty}}{(z ; q)_{\infty}}{ }_{2} \phi_{2}\left[\begin{array}{l}
a, c / b \\
c, a z
\end{array} ; b z\right] .
$$

This is a $q$-analogue of the classical relation

$$
{ }_{2} F_{1}\left[\begin{array}{c}
a, b \\
c
\end{array} ; z\right]=(1-z)^{-a}{ }_{2} F_{1}\left[\begin{array}{c}
a, c-b \\
c
\end{array} \frac{z}{z-1}\right] .
$$

Indeed, it can be shown, using the ${ }_{2} \mathcal{F}_{1}$ integral representation [19] or the defining set of $m$ partial differential equations [15, 24], that the multivariable analogue of $(5.2)$ holds in its full generality:

$$
{ }_{2} \mathcal{F}_{1}\left[\begin{array}{c}
a, b \\
c
\end{array} ; z\right]=\prod_{i=1}^{n}\left(1-z_{i}\right)^{-a}{ }_{2} \mathcal{F}_{1}\left[\begin{array}{c}
a, c-b \\
c
\end{array} ; \frac{z}{z-1}\right] .
$$

This is not possible in the multivariable version of (5.1); however, for the restricted argument $z t^{\delta}$, such a formula can be derived via a suitable modification of the argument in [7].

The key step is to establish the identity

$$
\prod_{i=1}^{n} \frac{\left(a z t^{n-i} ; q\right)_{\infty}}{\left(z t^{n-i} ; q\right)_{\infty}} \frac{(a ; q)_{\mu}}{\left(a z t^{n-1} ; q\right)_{\mu}} u_{0}\left(P_{\mu}\right)=\sum_{\lambda, \nu} t^{b(\nu)-b(\lambda)} \frac{(a ; q)_{\lambda}}{h_{\nu}^{\prime}} u_{0}\left(P_{\lambda}\right) z^{|\nu|} f_{\mu \nu}^{\lambda}
$$

for then it follows, from the Chu-Vandermonde formula (4.8), that

$$
\begin{aligned}
{ }_{2} \Phi_{1}\left[\begin{array}{c}
a, b \\
c
\end{array} ; z t^{\delta}\right] & =\sum_{\lambda, \mu, \nu}(-b)^{|\mu|} q^{b\left(\mu^{\prime}\right)} t^{b(\nu)-b(\lambda)} \frac{(c / b ; q)_{\mu}}{(c ; q)_{\mu} h_{\mu}^{\prime}} \frac{(a ; q)_{\lambda}}{h_{\lambda}^{\prime}} u_{0}\left(P_{\lambda}\right) z^{|\lambda|} f_{\mu \nu}^{\lambda} z^{|\mu|+|\nu|} \\
& =\prod_{i=1}^{n} \frac{\left(a z t^{n-i} ; q\right)_{\infty}}{\left(z t^{n-i} ; q\right)_{\infty}}{ }_{2} \Phi_{2}\left[\begin{array}{c}
a, c / b \\
c, a z t^{n-1} ; b z t^{\delta}
\end{array}\right]
\end{aligned}
$$

which is the multi-variable version of the Pfaff-Kummer transformation formula. It thus remains to prove (5.3). This identity is, in fact, a special case of the more general identity:

\section{Lemma 5.1.}

$$
\begin{aligned}
& \sum_{\sigma, \mu} \frac{(c ; q)_{\sigma} z^{|\sigma|}}{h_{\sigma}^{\prime}} \frac{(a ; q)_{\mu} x^{|\mu|} P_{\mu}\left(t^{\delta}\right)}{(b ; q)_{\mu}} f_{\lambda \sigma}^{\mu}(q, t) \\
& \quad=\prod_{i=1}^{n} \frac{\left(c z x t^{n-i} ; q\right)_{\infty}\left(a z x t^{n-i} ; q\right)_{\infty}}{\left(b t^{1-i} ; q\right)_{\infty}\left(z x t^{n-i} ; q\right)_{\infty}} \frac{(a x ; q)_{\lambda}}{\left(a z x t^{n-1} ; q\right)_{\lambda}} x^{|\lambda|} P_{\lambda}\left(t^{\delta}\right)
\end{aligned}
$$


provided $b=a c z x t^{n-1}$.

Proof. Let $S$ denote the summation side of the above equation. Note that from the definition of the generalized $q$-factorials and the $q$-binomial theorem, we can write

$$
\frac{(a ; q)_{\mu}}{(b ; q)_{\mu}}=\prod_{i=1}^{n} \frac{\left(a t^{1-i} ; q\right)_{\infty}}{\left(b t^{1-i} ; q\right)_{\infty}} \sum_{\tau} \frac{(b / a ; q)_{\tau}}{h_{\tau}^{\prime}}\left(a t^{1-n}\right)^{|\tau|} u_{\mu}\left(P_{\tau}(w)\right) .
$$

It follows that

$$
S=\prod_{i=1}^{n} \frac{\left(a t^{1-i} ; q\right)_{\infty}}{\left(b t^{1-i} ; q\right)_{\infty}} \sum_{\tau, \sigma, \mu} \frac{(c ; q)_{\sigma} z^{|\sigma|}}{h_{\sigma}^{\prime}} f_{\lambda \sigma}^{\mu}(q, t) x^{|\mu|}\left(a t^{1-n}\right)^{|\tau|} \frac{(b / a ; q)_{\tau}}{h_{\tau}^{\prime}} P_{\tau}\left(t^{\delta}\right) u_{\tau}\left(P_{\mu}(w)\right)
$$

where we also have used the symmetry property (1.10) for Macdonald polynomials. Now, from the definition of the coefficients $f_{\lambda \sigma}^{\mu}(q, t)$ and the $q$-binomial theorem, it also follows that

$$
\sum_{\sigma, \mu} \frac{(c ; q)_{\sigma} z^{|\sigma|}}{h_{\sigma}^{\prime}} f_{\lambda \sigma}^{\mu}(q, t) x^{|\mu|} u_{\tau}\left(P_{\mu}(w)\right)=u_{\tau}\left(\prod_{i=1}^{n} \frac{\left(c z x w_{i} ; q\right)_{\infty}}{\left(z x w_{i} ; q\right)_{\infty}} x^{|\lambda|} P_{\lambda}(w)\right) .
$$

Using this result in (5.5) gives

$$
\begin{aligned}
S= & \prod_{i=1}^{n} \frac{\left(a t^{1-i} ; q\right)_{\infty}\left(c z x t^{n-i} ; q\right)_{\infty}}{\left(b t^{1-i} ; q\right)_{\infty}\left(z x t^{n-i} ; q\right)_{\infty}} \\
& \times \sum_{\tau} \frac{\left(a t^{1-n}\right)^{|\tau|}(b / a ; q)_{\tau}\left(z x t^{n-1} ; q\right)_{\tau}}{h_{\tau}^{\prime}\left(c z x t^{n-1} ; q\right)_{\tau}} x^{|\lambda|} P_{\tau}\left(t^{\delta}\right) u_{\tau}\left(P_{\lambda}(w)\right) \\
= & \prod_{i=1}^{n} \frac{\left(a t^{1-i} ; q\right)_{\infty}\left(c z x t^{n-i} ; q\right)_{\infty}}{\left(b t^{1-i} ; q\right)_{\infty}\left(z x t^{n-i} ; q\right)_{\infty}} x^{|\lambda|} P_{\lambda}\left(t^{\delta}\right) \\
& \times \sum_{\tau} \frac{(b / a ; q)_{\tau}\left(z x t^{n-1} ; q\right)_{\tau}}{h_{\tau}^{\prime}\left(c z x t^{n-1} ; q\right)_{\tau}}\left(a t^{1-n}\right)^{|\tau|} u_{\lambda}\left(P_{\tau}(w)\right),
\end{aligned}
$$

where again, we have used the symmetry property (1.10). The crucial point now is that if $b=a c z x t^{n-1}$, then the sum over $\tau$ can be carried out using the $q$-binomial theorem again, i.e.,

$$
\sum_{\tau} u_{\lambda}\left(\frac{\left(z x t^{n-1} ; q\right)_{\tau}}{h_{\tau}^{\prime}} P_{\tau}\left(a t^{1-n} w\right)\right)=\prod_{i=1}^{n} \frac{\left(a z x t^{n-i} ; q\right)_{\infty}}{\left(a t^{1-i} ; q\right)_{\infty}} \frac{(a ; q)_{\lambda}}{\left(a z x t^{n-1} ; q\right)_{\lambda}}
$$

Using this in (5.6) gives the required result.

Remark 1. When $\lambda=0$, this identity reduces to the Gauss formula (2.4), and thus can be considered a "shifted" version of the Gauss theorem.

Remark 2. The identity (5.3) is simply the case $b=c=0, x=1$ of the above lemma.

5.1. Sear's ${ }_{4} \Phi_{3}$ transformation. A final application of the $q$-Saalschütz formulas (4.6) and (4.7) is in deriving a multivariable analogue of Sear's transformation of a terminating, balanced ${ }_{4} \phi_{3}$ series (see [7, eq. (3.2.1)]. Following the proof given in [7, Ex. 2.4], it suffices to write the product of two specific ${ }_{2} \Phi_{1}$ series in two different ways 
and compare coefficients of the Macdonald polynomial $P_{\left(N^{n}\right)}(z)$ in each case. Indeed, the coefficient of $P_{\left(N^{n}\right)}(z)$ in the product

$$
P:={ }_{2} \Phi_{1}\left[\begin{array}{c}
a, b \\
c
\end{array} ; z\right]{ }_{2} \Phi_{1}\left[\begin{array}{c}
d, e \\
a b d e / c
\end{array} ; \frac{a b z}{c}\right]
$$

can be seen to be

$$
\frac{(a ; q)_{\left(N^{n}\right)}(b ; q)_{\left(N^{n}\right)}}{(c ; q)_{\left(N^{n}\right)} h_{\left(N^{n}\right)}^{\prime}} \Phi_{3}\left[\begin{array}{c}
q^{-N}, t^{n-1} q^{1-N} c^{-1}, d, e \\
t^{n-1} q^{1-N} a^{-1}, t^{n-1} q^{1-N} b^{-1}, a b d e / c
\end{array} ; q t^{\delta}\right]
$$

where one must use the expression for $f_{\mu \nu}^{\left(N^{n}\right)}(q, t)$ given in (4.2), along with (4.1) and (4.5). On the other hand, we have by Euler's transformation (3.2):

$$
P=\prod_{i=1}^{n} \frac{\left(z_{i} ; q\right)_{\infty}}{\left(a b z_{i} / c ; q\right)_{\infty}}{ }_{2} \Phi_{1}\left[\begin{array}{c}
a b e / c, a b d / c \\
a b d e / c
\end{array} ; z\right]{ }_{2} \Phi_{1}\left[\begin{array}{c}
a, b \\
c
\end{array} ; z\right]
$$

Expanding this using the $q$-binomial theorem and then examining the coefficient of $P_{\left(N^{n}\right)}(z)$, it follows from use of $(4.1),(4.2),(4.5)$ and (4.6) that the coefficient of $P_{\left(N^{n}\right)}(z)$ in $(5.8)$ is

$$
\left(\frac{a b}{c}\right)^{n N} \frac{(c / a ; q)_{\left(N^{n}\right)}(c / b ; q)_{\left(N^{n}\right)}}{(c ; q)_{\left(N^{n}\right)} h_{\left(N^{n}\right)}^{\prime}} \Phi_{3}\left[\begin{array}{c}
q^{-N}, t^{n-1} q^{1-N} c^{-1}, a b e / c, a b d / c \\
t^{n-1} q^{1-N} a c^{-1}, t^{n-1} q^{1-N} b c^{-1}, a b d e / c^{\prime}
\end{array} ; t^{\delta}\right] .
$$

Comparing (5.7) and (5.9) yields the multivariable analogue of Sear's transformation

$$
\begin{aligned}
& { }_{4} \Phi_{3}\left[\begin{array}{c}
q^{-N}, t^{n-1} q^{1-N} c^{-1}, d, e \\
t^{n-1} q^{1-N} a^{-1}, t^{n-1} q^{1-N} b^{-1}, a b d e / c
\end{array} ; t^{\delta}\right]=\left(\frac{a b}{c}\right)^{n N} \\
& \times \frac{(c / a ; q)_{\left(N^{n}\right)}(c / b ; q)_{\left(N^{n}\right)}}{(a ; q)_{\left(N^{n}\right)}(b ; q)_{\left(N^{n}\right)}}{ }_{4} \Phi_{3}\left[\begin{array}{c}
q^{-N}, t^{n-1} q^{1-N} c^{-1}, a b e / c, a b d / c \\
\left.t^{n-1} q^{1-N} a c^{-1}, t^{n-1} q^{1-N} b c^{-1}, a b d e / c^{;} q t^{\delta}\right] .
\end{array}\right.
\end{aligned}
$$

\section{Bilateral series}

We now turn our attention to bilateral basic hypergeometric series. The key point is that by using the following property of the Macdonald polynomials [20]

$$
|x|^{a} P_{\lambda}(x)=P_{\lambda+a}(x), \quad a \in \mathbb{Z}, \quad|x|:=x_{1} \cdots x_{n},
$$

where $\lambda+a:=\left(\lambda_{1}+a, \ldots, \lambda_{n}+a\right)$, one can define Macdonald polynomials for all $n$-tuples $\lambda$ with $\lambda_{1} \geq \lambda_{2} \geq \cdots \geq \lambda_{n}$ where $\lambda_{i} \in \mathbb{Z}$, namely, if $\lambda_{n}<0$, then $P_{\lambda}(x)=$ $|x|^{\lambda_{n}} P_{\lambda-\lambda_{n}}(x)$. Denote the set of such partitions as $\mathcal{P}$ and those with nonnegative entries as $\mathcal{P}_{+}$.

It also follows from (6.1) and the definition of the inner product on the space of Macdonald polynomials that, for any $\lambda \in \mathcal{P}_{+}$and any $a$, we have $\left\langle|x|^{a} P_{\lambda}, P_{\mu}\right\rangle_{k}=$ $\left\langle P_{\lambda},|x|^{-a} P_{\mu}\right\rangle_{k}$, and it follows that we can extend the definition of the inner product to polynomials $P_{\lambda}$ for all $\lambda \in \mathcal{P}$, and that they remain orthogonal, i.e.,

$$
\left\langle P_{\lambda}, P_{\mu}\right\rangle_{k}=\delta_{\lambda \mu}\left\langle P_{\lambda-\lambda_{n}}, P_{\lambda-\lambda_{n}}\right\rangle_{k}
$$


Following Kaneko [14], define the multivariable bilateral hypergeometric series by

$$
\begin{aligned}
{ }_{r} \Psi_{s+1}\left[\begin{array}{c}
a_{1}, \ldots, a_{r} \\
b, b_{1}, \ldots, b_{s}
\end{array} ; z\right]:=\prod_{i=1}^{n} \frac{\left(b t^{i-1} ; q\right)_{\infty}(q ; q)_{\infty}}{\left(q t^{i-1} ; q\right)_{\infty}(b ; q)_{\infty}} \\
\quad \times \sum_{\lambda \in \mathcal{P}}\left((-1)^{|\lambda|} q^{n\left(\lambda^{\prime}\right)}\right)^{s+1-r} \frac{\left(q t^{n-1} ; q\right)_{\lambda}\left(a_{1} ; q\right)_{\lambda} \cdots\left(a_{r} ; q\right)_{\lambda}}{\left(b t^{n-1} ; q\right)_{\lambda}\left(b_{1} ; q\right)_{\lambda} \cdots\left(b_{s} ; q\right)_{\lambda} h_{\lambda}^{\prime}} P_{\lambda}(z) .
\end{aligned}
$$

For $\lambda_{n}<0$, the above factorial symbols $(a)_{\lambda}$ must be interpreted according to

$$
(a ; q)_{\lambda}:=t^{\sum_{i}(i-1) \lambda_{i}} \prod_{i=1}^{n} \frac{\left(a t^{1-i} ; q\right)_{\infty}}{\left(a q^{\lambda_{i}} t^{1-i} ; q\right)_{\infty}}
$$

and $h_{\lambda}^{\prime}$ is interpreted according to (4.4).

6.1. Kaneko's ${ }_{1} \Psi_{1}$ summation formula. In [14], Kaneko gave a simple argument for the summation of a ${ }_{1} \Psi_{1}$ series, following Ismail's argument for the one-variable case [10]. Here, we give an alternative proof following Andrew's argument in the one-variable case [1] which uses the Gauss theorem.

Theorem 6.1 (Kaneko). For $|b / a|<\left|x_{i}\right|<1$ for all $i=1, \ldots, n$, we have

$$
{ }_{1} \Psi_{1}\left[\begin{array}{l}
a \\
b
\end{array} ; x\right]=\prod_{i=1}^{n} \frac{\left(a x_{i} ; q\right)_{\infty}\left(q / a x_{i} ; q\right)_{\infty}\left(b t^{i-1} / a ; q\right)_{\infty}(q ; q)_{\infty}}{\left(x_{i} ; q\right)_{\infty}\left(b / a x_{i} ; q\right)_{\infty}\left(q t^{i-1} / a ; q\right)_{\infty}(b ; q)_{\infty}} .
$$

Proof. We have from the $q$-binomial theorem that

$$
\begin{aligned}
\prod_{i=1}^{n} \frac{\left(b / a x_{i} ; q\right)_{\infty}}{\left(q / a x_{i} ; q\right)_{\infty}}{ }_{1} \Psi_{1}\left[\begin{array}{l}
a \\
b
\end{array} ; x\right]= & \prod_{i=1}^{n} \frac{\left(b t^{i-1} ; q\right)_{\infty}(q ; q)_{\infty}}{\left(q t^{i-1} ; q\right)_{\infty}(b ; q)_{\infty}} \\
& \times \sum_{\substack{\sigma \in \mathcal{P}_{+} \\
\mu \in \mathcal{P}}} \frac{(b / q ; q)_{\sigma}}{h_{\sigma}^{\prime}}\left(\frac{q}{a}\right)^{|\sigma|} \frac{\left(q t^{n-1} ; q\right)_{\mu}}{\left(b t^{n-1} ; q\right)_{\mu} h_{\mu}^{\prime}} P_{\sigma}\left(x^{-1}\right) P_{\mu}(x) .
\end{aligned}
$$

We need to expand $P_{\sigma}\left(x^{-1}\right) P_{\mu}(x)$ in terms of Macdonald polynomials. Suppose

$$
P_{\sigma}\left(x^{-1}\right) P_{\mu}(x)=\sum_{\lambda \in \mathcal{P}} d_{\sigma \mu}^{\lambda} P_{\lambda}(x) .
$$

From the orthogonality of $\left\{P_{\lambda}\right\}_{\lambda \in \mathcal{P}}$, we have that

$$
\begin{aligned}
\left\langle P_{\nu-\nu_{n}}(x), P_{\nu-\nu_{n}}(x)\right\rangle_{k} d_{\sigma \mu}^{\nu} & =\left\langle P_{\mu}(x), P_{\sigma}\left(x^{-1}\right) P_{\mu}(x)\right\rangle_{k} \\
& =\left\langle P_{\nu-\nu_{n}}(x) P_{\sigma}(x), P_{\mu-\nu_{n}}(x)\right\rangle_{k} .
\end{aligned}
$$

Since both $\sigma$ and $\nu-\nu_{n} \in \mathcal{P}_{+}$, then $d_{\sigma \mu}^{\nu}$ is nonzero only for all $\mu$ such that $\mu-\nu_{n} \in \mathcal{P}_{+}$. Thus,

$$
d_{\sigma \mu}^{\nu}=\frac{\left\langle P_{\mu-\nu_{n}}, P_{\mu-\nu_{n}}\right\rangle_{k}}{\left\langle P_{\nu-\nu_{n}}, P_{\nu-\nu_{n}}\right\rangle_{k}} f_{\nu-\nu_{n}, \sigma}^{\mu-\nu_{n}}=\frac{h_{\mu-\nu_{n}}^{\prime} h_{\nu-\nu_{n}}}{h_{\mu-\nu_{n}} h_{\nu-\nu_{n}}^{\prime}} \frac{\left(t^{n} ; q\right)_{\mu-\nu_{n}}\left(q t^{n-1} ; q\right)_{\nu-\nu_{n}}}{\left(q t^{n-1} ; q\right)_{\mu-\nu_{n}}\left(t^{n} ; q\right)_{\nu-\nu_{n}}} f_{\nu-\nu_{n}, \sigma}^{\mu}
$$


Thus, we have

$$
\begin{aligned}
\prod_{i=1}^{n} \frac{\left(b / a x_{i} ; q\right)_{\infty}(b ; q)_{\infty}}{\left(q / a x_{i} ; q\right)_{\infty}(q ; q)_{\infty}}{ }_{1} \Psi_{1}\left[\begin{array}{l}
a \\
b
\end{array} ; x\right] \\
=\prod_{i=1}^{n} \frac{\left(b t^{i-1} ; q\right)_{\infty}}{\left(q t^{i-1} ; q\right)_{\infty}} \sum_{\nu \in \mathcal{P}} \frac{h_{\nu-\nu_{n}}\left(q t^{n-1} ; q\right)_{\nu-\nu_{n}}}{h_{\nu-\nu_{n}}^{\prime}\left(t^{n} ; q\right)_{\nu-\nu_{n}}} B_{\nu} P_{\nu}(x)
\end{aligned}
$$

where

$$
\begin{aligned}
B_{\nu}=\prod_{i=1}^{n} \frac{\left(b q^{\nu_{n}} t^{n-i} ; q\right)_{-\nu_{n}}}{\left(a q^{\nu_{n}} t^{1-i} ; q\right)_{-\nu_{n}}} \sum_{\sigma \in \mathcal{P}_{+}} \sum_{\substack{\mu \text { s.t. } \\
\mu-\nu_{n} \in \mathcal{P}_{+}}} \frac{(b / q ; q)_{\sigma}}{h_{\sigma}^{\prime}}\left(\frac{q}{a}\right)^{|\sigma|} \\
\quad \times \frac{\left(a q^{\nu_{n}} ; q\right)_{\mu-\nu_{n}}}{\left(b q^{\nu_{n}} t^{n-1} ; q\right)_{\mu-\nu_{n}}} P_{\mu-\nu_{n}}\left(t^{\delta}\right) f_{\nu-\nu_{n}, \sigma}^{\mu-\nu_{n}}(q, t) .
\end{aligned}
$$

We now observe that we can use Lemma 5.1 to sum the above expression, yielding

$B_{\nu}=\prod_{i=1}^{n} \frac{\left(b q^{\nu_{n}} t^{n-i} ; q\right)_{-\nu_{n}}\left(b t^{i-1} / a ; q\right)_{\infty}\left(q^{\nu_{n}+1} t^{n-i} ; q\right)_{\infty}}{\left(a q^{\nu_{n}} t^{1-i} ; q\right)_{-\nu_{n}}\left(q t^{i-1} ; q\right)_{\infty}\left(q t^{n-i} / a ; q\right)_{\infty}} \frac{\left(a q^{\nu_{n}} ; q\right)_{\nu-\nu_{n}}}{\left(q^{\nu_{n}+1} t^{n-1} ; q\right)_{\nu-\nu_{n}}} P_{\nu-\nu_{n}}\left(t^{\delta}\right)$.

Clearly $B_{\nu}=0$ when $\nu_{n}<0$. In the cases when $\nu_{n} \geq 0$ (i.e., $\nu \in \mathcal{P}_{+}$), it follows after some manipulations that

$$
B_{\nu}=\prod_{i=1}^{n} \frac{\left(b t^{i-1} / a ; q\right)_{\infty}\left(q t^{i-1} ; q\right)_{\infty}}{\left(q t^{i-1} / a ; q\right)_{\infty}\left(b t^{i-1} ; q\right)_{\infty}} \frac{h_{\nu-\nu_{n}}^{\prime}\left(t^{n} ; q\right)_{\nu-\nu_{n}}}{h_{\nu-\nu_{n}}\left(q t^{n-1} ; q\right)_{\nu-\nu_{n}}} \frac{(a ; q)_{\nu}}{h_{\nu}^{\prime}}
$$

Substituting back into (6.4) and using the $q$-binomial theorem gives the result.

6.2. ${ }_{2} \Psi_{2}$ transformations. Our final application of the theory of Macdonald polynomials will be to transformation and summation formulas for bilateral ${ }_{2} \Psi_{2}$ series. We begin with the following important result of Kadell and Kaneko [13, 16, 18] which is equivalent to the ${ }_{1} \Psi_{1}$ summation theorem given above.

Theorem 6.2. Given a partition $\lambda \in \mathcal{P}$, let

$$
A_{\lambda}(a, b):=\text { C.T. }\left\{P_{\lambda}(x) \prod_{i=1}^{n}\left(x_{i} ; q\right)_{a}\left(q / x_{i} ; q\right)_{b} \Delta_{k}(x)\right\}
$$

where $\Delta_{k}(x)$ is the Macdonald weight function given in (2.2) with $t=q^{k}, k$ a positive integer, and $a, b$ are arbitrary complex numbers. Then

$$
A_{\lambda}(a, b)=q^{(1+b)|\lambda|} \prod_{i=1}^{n} \frac{\left(q^{1+a} t^{i-1} ; q\right)_{\infty}\left(q^{1+b} t^{i-1} ; q\right)_{\infty}}{\left(q t^{i-1} ; q\right)_{\infty}\left(q^{1+a+b} t^{i-1} ; q\right)_{\infty}} \frac{\left(q^{-b} ; q\right)_{\lambda}}{\left(q^{1+a} t^{n-1} ; q\right)_{\lambda}} P_{\lambda}\left(t^{\delta}\right)\langle 1,1\rangle_{q, q^{k}}
$$

We remark that here there is nothing essential about the restriction $t=q^{k}$ in Theorem 7.2; the result remains valid for general $t$ provided C.T. is interpreted as an appropriate contour integral.

The connection with the ${ }_{1} \Psi_{1}$ formula is as follows: suppose

$$
\prod_{i=1}^{n}\left(x_{i} ; q\right)_{a}\left(q / x_{i} ; q\right)_{b}=\sum_{\lambda \in \mathcal{P}} c_{\lambda}(a, b) P_{\lambda}\left(x^{-1}\right) \text {. }
$$


It follows from the orthogonality of Macdonald polynomials that $c_{\lambda}(a, b)=A_{\lambda}(a, b) /$ $\left\langle P_{\lambda}, P_{\lambda}\right\rangle_{k}$ with $A_{\lambda}(a, b)$ given as in Theorem 6.2. Now, the transformation $x_{i} \rightarrow q^{-u} / x_{i}$ certainly doesn't affect the constant term of the expression appearing in Theorem 6.2. Thus

$$
\text { C.T. }\left\{P_{\lambda}\left(x^{-1}\right) \prod_{i=1}^{n}\left(q^{-u} / x_{i} ; q\right)_{a^{\prime}}\left(q^{1+u} x_{i} ; q\right)_{b^{\prime}} \Delta_{k}(x)\right\}=q^{u|\lambda|} A_{\lambda}\left(a^{\prime}, b^{\prime}\right) .
$$

As above, it follows that if

$$
\prod_{i=1}^{n}\left(q^{-u} / x_{i} ; q\right)_{a^{\prime}}\left(q^{1+u} x_{i} ; q\right)_{b^{\prime}}=\sum_{\mu \in \mathcal{P}} c_{\mu}^{\prime}\left(a^{\prime}, b^{\prime}\right) P_{\mu}(x)
$$

then $c_{\mu}^{\prime}\left(a^{\prime}, b^{\prime}\right)=q^{u|\mu|} A_{\mu}\left(a^{\prime}, b^{\prime}\right) /\left\langle P_{\mu}, P_{\mu}\right\rangle_{k}$. Define

$$
I\left(a, b, a^{\prime}, b^{\prime} ; u\right):=\text { C.T. }\left\{\prod_{i=1}^{n}\left(x_{i} ; q\right)_{a}\left(q / x_{i} ; q\right)_{b}\left(q^{-u} / x_{i} ; q\right)_{a^{\prime}}\left(q^{u+1} x_{i} ; q\right)_{b^{\prime}} \Delta_{k}(x)\right\} \text {. }
$$

It follows from (6.5) and (6.7) that

$$
\begin{aligned}
I\left(a, b, a^{\prime}, b^{\prime} ; u\right)= & \sum_{\lambda \in \mathcal{P}} c_{\lambda}(a, b) c_{\lambda}^{\prime}\left(a^{\prime}, b^{\prime}\right)\left\langle P_{\lambda}, P_{\lambda}\right\rangle_{k} \\
= & \prod_{i=1}^{n} \frac{\left(q^{1+a} ; q\right)_{\infty}\left(q^{1+b} t^{i-1} ; q\right)_{\infty}\left(q^{1+a^{\prime}} t^{i-1} ; q\right)_{\infty}\left(q^{1+b^{\prime}} t^{i-1} ; q\right)_{\infty}}{(q ; q)_{\infty}\left(q t^{i-1} ; q\right)_{\infty}\left(q^{1+a+b} t^{i-1} ; q\right)_{\infty}\left(q^{1+a^{\prime}+b^{\prime}} t^{i-1} ; q\right)_{\infty}}\langle 1,1\rangle_{q, q^{k}} \\
& \times{ }_{2} \Psi_{2}\left[\begin{array}{c}
q^{-b}, q^{-b^{\prime}} \\
q^{1+a}, q^{1+a^{\prime}} t^{n-1}
\end{array} q^{b+b^{\prime}+2+u} t^{\delta}\right] .
\end{aligned}
$$

We now note that there are a couple of values of $u$ for which we can derive an alternative expression for $I\left(a, b, a^{\prime}, b^{\prime} ; u\right)$.

The case $u=a-1$. In this case, note that $\left(x_{i} ; q\right)_{a}\left(q^{a} x_{i} ; q\right)_{b^{\prime}}=\left(x_{i} ; q\right)_{a+b^{\prime}}$. Using the $q$-binomial theorem to expand $\prod_{i}\left(q^{-u} / x_{i} ; q\right)_{a^{\prime}}$ and inserting in (6.8), we have

$$
\begin{aligned}
I & =\sum_{\lambda \in \mathcal{P}_{+}} \frac{\left(q^{-a^{\prime}} ; q\right)_{\lambda}}{h_{\lambda}^{\prime}} q^{\left(a^{\prime}+1-a\right)|\lambda|} \text { C.T. }\left\{\prod_{i=1}^{n}\left(x_{i} ; q\right)_{a+b^{\prime}}\left(q / x_{i} ; q\right)_{b} P_{\lambda}\left(x^{-1}\right) \Delta_{k}(x)\right\} \\
& =\prod_{i=1}^{n} \frac{\left(q^{1+b} t^{i-1} ; q\right)_{\infty}\left(q^{1+a+b^{\prime}} t^{i-1} ; q\right)_{\infty}}{\left(q t^{i-1} ; q\right)_{\infty}\left(q^{1+a+b+b^{\prime}} t^{i-1} ; q\right)_{\infty}}\langle 1,1\rangle_{k 2} \Phi_{1}\left[\begin{array}{c}
q^{-a^{\prime}}, q^{-a-b^{\prime}} \\
q^{1+b} t^{n-1}
\end{array} ; q^{1+a^{\prime}+b^{\prime}} t^{\delta}\right]
\end{aligned}
$$

where we have used (6.6) with $u=-1$. Comparing (6.10) with the $u=a-1$ case of (6.9) gives (upon setting $\alpha_{1}=q^{-b}, \alpha_{2}=q^{-b^{\prime}}, \alpha_{3}=q^{1+a}$, and $\alpha_{4}=q^{1+a^{\prime}}$ ) the transformation formula

$$
\begin{aligned}
{ }_{2} \Psi_{2}\left[\begin{array}{c}
\alpha_{1}, \alpha_{2} \\
\alpha_{3}, \alpha_{4} t^{n-1} ;
\end{array} \frac{\alpha_{3}}{\alpha_{1} \alpha_{2}} t^{\delta}\right] \\
=\prod_{i=1}^{n} \frac{(q ; q)_{\infty}\left(\alpha_{3} t^{i-1} / \alpha_{1} ; q\right)_{\infty}\left(\alpha_{4} t^{i-1} / \alpha_{2} ; q\right)_{\infty}\left(\alpha_{3} t^{i-1} / \alpha_{2} ; q\right)_{\infty}}{\left(\alpha_{3} ; q\right)_{\infty}\left(\alpha_{4} t^{i-1} ; q\right)_{\infty}\left(q t^{i-1} / \alpha_{2} ; q\right)_{\infty}\left(\alpha_{3} t^{i-1} / \alpha_{1} \alpha_{2} ; q\right)_{\infty}} \\
\quad \times{ }_{2} \Phi_{1}\left[\begin{array}{c}
q / \alpha_{4}, q \alpha_{2} / \alpha_{3} \\
q t^{n-1} / \alpha_{1}
\end{array} \frac{\alpha_{4}}{\alpha_{2}} t^{\delta}\right] .
\end{aligned}
$$


Note that when $\alpha_{3}=q \alpha_{1}$, the ${ }_{2} \Phi_{1}$ appearing in the above equation can be summed by the Gauss formula (2.4) yielding the summation formula

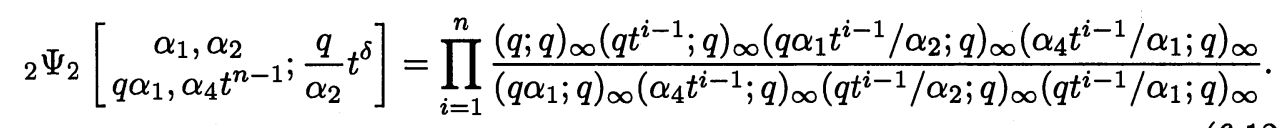

The case $u=-1$. Note from the definition (6.8) that

$$
I\left(a, b, a^{\prime}, b^{\prime} ;-1\right)=I\left(b^{\prime}, b, a^{\prime}, a ;-1\right)=I\left(a, a^{\prime}, b, b^{\prime} ;-1\right) .
$$

Taking, for example, the first of these relations, and using the equivalent expressions given by (6.9) gives the following transformation formula:

$$
\begin{aligned}
{ }_{2} \Psi_{2}\left[\begin{array}{c}
\alpha_{1}, \alpha_{2} \\
\left.\alpha_{3}, \alpha_{4} t^{n-1} ; \frac{q}{\alpha_{1} \alpha_{2}} t^{\delta}\right]
\end{array}\right. \\
=\prod_{i=1}^{n} \frac{\left(q / \alpha_{2} ; q\right)_{\infty}\left(\alpha_{3} t^{i-1} ; q\right)_{\infty}\left(\alpha_{4} t^{i-1} / \alpha_{2} ; q\right)_{\infty}\left(\alpha_{3} t^{i-1} / \alpha_{1} ; q\right)_{\infty}}{\left(\alpha_{3} ; q\right)_{\infty}\left(q t^{i-1} / \alpha_{2} ; q\right)_{\infty}\left(q t^{i-1} / \alpha_{1} \alpha_{2} ; q\right)_{\infty}\left(\alpha_{3} \alpha_{4} t^{i-1} / q ; q\right)_{\infty}} \\
\quad \times{ }_{2} \Psi_{2}\left[\begin{array}{c}
\alpha_{1}, q \alpha_{3} \\
\left.q / \alpha_{2}, \alpha_{4} t^{n-1} ; \frac{\alpha_{3}}{\alpha_{1}} t^{\delta}\right] .
\end{array}\right.
\end{aligned}
$$

6.3. The $q \rightarrow 1^{-}$limit. One of the curious features of the bilateral basic series ${ }_{2} \psi_{2}$ is that there appears to be no analogue of the Gauss summation for the ${ }_{2} \phi_{1}$ series, whereas in the $q=1$ case, the bilateral ${ }_{2} H_{2}$ series with unit argument indeed can be summed [6]. Let us now show that this behavior carries over in the multivariable case. We begin by defining the limit as $q$ tends to one from below of the bilateral series ${ }_{r} \Psi_{s+1}$, namely,

$$
\begin{aligned}
{ }_{r} H_{s+1}\left[\begin{array}{c}
a_{1} \ldots, a_{r} \\
b, b_{1} \ldots, b_{s}
\end{array} ;\right]:=\prod_{i=1}^{n} & \frac{\Gamma(1+k(i-1)) \Gamma(b)}{\Gamma(b+k(i-1))} \\
& \times \sum_{\lambda \in \mathcal{P}} \frac{(1+k(n-1))_{\lambda}\left(a_{1}\right)_{\lambda} \cdots\left(a_{r}\right)_{\lambda}}{(b+k(n-1))_{\lambda}\left(b_{1}\right)_{\lambda} \cdots\left(b_{s}\right)_{\lambda} d_{\lambda}^{\prime}} P_{\lambda}(x ; k) .
\end{aligned}
$$

Here $P_{\lambda}(x ; k)$ is the Jack polynomial (normalized so that the coefficient of the monomial symmetric function $m_{\lambda}(x)$ is unity), the generalized Pochhammer symbols are defined by $(a)_{\lambda}:=\prod_{i}(a+k(1-i))_{\lambda_{i}}$ with $(a)_{n}:=a(a+1) \cdots(a+n-1)$ being the usual Pochhammer symbol, and $d_{\lambda}^{\prime}:=\prod_{s \in \lambda}(a(a)+1+k l(s))$ which is the limit as $q$ tends to one from below of $h_{\lambda}^{\prime}$ defined in (2.3).

It follows from (6.9) that

$$
\begin{aligned}
\lim _{q \rightarrow 1^{-}} & I\left(a, b, a^{\prime}, b^{\prime} ; u\right) \\
= & \prod_{i=1}^{n} \frac{\Gamma(1+k(i-1)) \Gamma(1+a+b+k(i-1)) \Gamma\left(1+a^{\prime}+b^{\prime}+k(i-1)\right)}{\Gamma(1+a) \Gamma(1+b+k(i-1)) \Gamma\left(1+b^{\prime}+k(i-1)\right) \Gamma\left(1+a^{\prime}+k(i-1)\right)} \\
& \quad \times \frac{(k n) !}{n !(k !)^{n}}{ }_{2} H_{2}\left[\begin{array}{c}
-b,-b^{\prime} \\
1+a, 1+a^{\prime}+k(n-1)
\end{array} ; 1^{n}\right]
\end{aligned}
$$

where we have used the fact that

$$
\lim _{q \rightarrow 1^{-}}\langle 1,1\rangle_{q, q^{k}}=\frac{1}{n !} \frac{(k n) !}{(k !)^{n}}
$$


which follows from (1.7).

The crucial point now is that in the limit $q \rightarrow 1^{-}$, we can combine the quantities $a$ and $b^{\prime}$ (respectively, $a^{\prime}$ and $b$ ) in the definition of $I\left(a, b, a^{\prime}, b^{\prime} ; u\right)$ in (6.8), so that

$$
\begin{aligned}
\lim _{q \rightarrow 1^{-}} I\left(a, b, a^{\prime}, b^{\prime} ; u\right) & =\lim _{q \rightarrow 1^{-}} A_{0}\left(a+b^{\prime}, a^{\prime}+b\right) \\
& =\frac{(k n) !}{n !(k !)^{n}} \prod_{i=1}^{n} \frac{\Gamma(1+k(i-1)) \Gamma\left(1+a+a^{\prime}+b+b^{\prime}+k(i-1)\right)}{\Gamma\left(1+a+b^{\prime}+k(i-1)\right) \Gamma\left(1+a^{\prime}+b+k(i-1)\right)} .
\end{aligned}
$$

From these two equations, we have the summation formula

$$
\begin{aligned}
{ }_{2} H_{2} & {\left[\begin{array}{c}
-b,-b^{\prime} \\
1+a, 1+a^{\prime}+k(n-1)
\end{array} 1^{n}\right] } \\
= & \prod_{i=1}^{n} \frac{\Gamma(1+a) \Gamma\left(1+a+a^{\prime}+b+b^{\prime}+k(i-1)\right)}{\Gamma\left((1+a+b+k(i-1)) \Gamma\left(1+a^{\prime}+b^{\prime}+k(i-1)\right)\right.} \\
& \quad \times \prod_{i=1}^{n} \frac{\Gamma(1+b+k(i-1)) \Gamma\left(1+b^{\prime}+k(i-1)\right) \Gamma\left(1+a^{\prime}+k(i-1)\right)}{\Gamma\left(1+a+b^{\prime}+k(i-1)\right) \Gamma\left(1+a^{\prime}+b+k(i-1)\right)} .
\end{aligned}
$$

which certainly reduces to Dougall's result [6] when $n=1$.

6.4. Bailey's ${ }_{2} \Psi_{2}$ transformations. In this final section, we shall derive multivariable analogues of Bailey's general transformations for ${ }_{2} \psi_{2}$ series [3]. First, given a general partition $\lambda \in \mathcal{P}$, define $-\lambda^{R}:=\left(-\lambda_{n},-\lambda_{n-1}, \ldots,-\lambda_{1}\right)$. It follows from (4.3) (which can easily be seen to hold for all partitions $\sigma \in \mathcal{P}$ ) and (6.1) that

$$
P_{-\lambda^{R}}(z)=P_{\lambda}\left(z^{-1}\right), \quad \text { for } \lambda \in \mathcal{P} .
$$

It also follows from the definitions that

$$
(a ; q)_{-\lambda^{R}}=\frac{(-q / a)^{|\lambda|} q^{n\left(\lambda^{\prime}\right)}}{\left(q t^{n-1} / a ; q\right)_{\lambda}} \quad \frac{\left(q t^{n-1} ; q\right)_{-\lambda^{R}}}{h_{-\lambda^{R}}^{\prime}}=t^{(1-n)|\lambda|} \frac{\left(q t^{n-1} ; q\right)_{\lambda}}{h_{\lambda}^{\prime}} .
$$

The proof follows [3]: from the ${ }_{1} \Psi_{1}$ summation formula (6.3), it follows that

$$
\begin{aligned}
& { }_{1} \Psi_{1}\left[\begin{array}{l}
b \\
c^{\prime}
\end{array} a z\right]{ }_{1} \Psi_{1}\left[\begin{array}{l}
a b^{\prime} \\
c^{\prime}
\end{array} ;\right] \\
& =\prod_{i=1}^{n} \frac{\left(c t^{i-1} / b ; q\right)_{\infty}\left(c^{\prime} t^{i-1} / a b^{\prime} ; q\right)_{\infty}\left(q t^{i-1} / b^{\prime} ; q\right)_{\infty}\left(q t^{i-1} / a b ; q\right)_{\infty}}{\left(q t^{i-1} / b ; q\right)_{\infty}\left(q t^{i-1} / a b^{\prime} ; q\right)_{\infty}\left(c^{\prime} t^{i-1} / b^{\prime} ; q\right)_{\infty}\left(c t^{i-1} / a b ; q\right)_{\infty}} \\
& \times{ }_{1} \Psi_{1}\left[\begin{array}{l}
b^{\prime} \\
c^{\prime}
\end{array} ; a z\right]{ }_{1} \Psi_{1}\left[\begin{array}{c}
a b \\
c
\end{array} ;\right] .
\end{aligned}
$$

We now need to compare the constant terms on each side. Certainly, the product $P_{\lambda}(z) P_{\mu}(z)$ only has a constant term if $\mu=-\lambda^{R}$ and that constant term is $\left\langle P_{\lambda}, P_{\lambda}\right\rangle_{k} /\langle 1,1\rangle_{k}$. If we now use this fact to write down the constant terms in each side of (6.18)), using the simplifications (6.17) and changing parameters, we end up 
with the transformation

$$
\begin{aligned}
{ }_{2} \Psi_{2}\left[\begin{array}{c}
\alpha_{1}, \alpha_{2} \\
\alpha_{3}, \alpha_{4} t^{n-1} ; z t^{\delta}
\end{array}\right] \\
=\prod_{i=1}^{n} \frac{\left(\alpha_{3} t^{i-1} / \alpha_{1} ; q\right)_{\infty}\left(\alpha_{4} t^{i-1} / \alpha_{2} ; q\right)_{\infty}\left(\alpha_{2} z t^{i-1} ; q\right)_{\infty}\left(q \alpha_{4} t^{i-1} / \alpha_{1} \alpha_{2} z ; q\right)_{\infty}}{\left(q t^{i-1} / \alpha_{1} ; q\right)_{\infty}\left(\alpha_{4} t^{i-1} ; q\right)_{\infty}\left(z t^{i-1} ; q\right)_{\infty}\left(\alpha_{3} \alpha_{4} t^{i-1} / \alpha_{1} \alpha_{2} z ; q\right)_{\infty}} \\
\quad \times \prod_{i=1}^{n} \frac{\left(\alpha_{3} t^{i-1} ; q\right)_{\infty}\left(q / \alpha_{2} ; q\right)_{\infty}}{\left(q t^{i-1} / \alpha_{2} ; q\right)_{\infty}\left(\alpha_{3} ; q\right)_{\infty}}{ }_{2} \Psi_{2}\left[\begin{array}{c}
q / \alpha_{2} z, q / \alpha_{3} \\
q / \alpha_{2}, q t^{n-1} \alpha_{4} / \alpha_{1} \alpha_{2} z
\end{array} ; \frac{\alpha_{3}}{\alpha_{1}} t^{\delta}\right] .
\end{aligned}
$$

Following [3], we can rewrite this using the identity

$$
{ }_{2} \Psi_{2}\left[\begin{array}{l}
a, b \\
c, d
\end{array} ; z\right]=\prod_{i=1}^{n} \frac{\left(c t^{i-1} ; q\right)_{\infty}(q / a ; q)_{\infty}}{(c ; q)_{\infty}\left(q t^{i-1} / a ; q\right)_{\infty}}{ }_{2} \Psi_{2}\left[\begin{array}{l}
q / c, q t^{n-1} / d \\
q / a, q t^{n-1} / b
\end{array} ; \frac{c d}{a c} z^{-1}\right],
$$

which follows from making the substitution $\lambda \rightarrow-\lambda^{R}$ in the definition of the ${ }_{2} \Psi_{2}$ series and simplifying using (6.17). The final result is thus

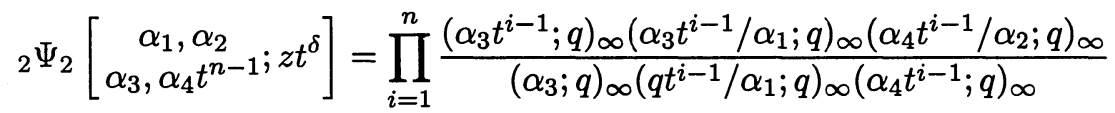

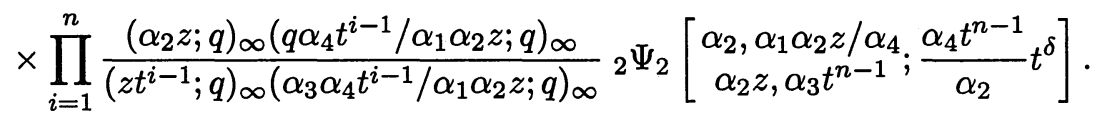

We remark here that the transformations appearing in previous section (6.11), (6.13) are special cases of the transformation (6.19).

Acknowledgment. The authors thank George Andrews for discussions, and the referee for helpful comments. They acknowledge the financial support of the Australian Research Council.

\section{References}

1. G. E. Andrews, On Ramanujan's summation of ${ }_{1} \psi_{1}(a ; b ; z)$, Proc. Amer. Math. Soc. 22 (1969), $522-523$.

2. R. Askey, Some basic hypergeometric extensions of integrals of Selberg and Andrews, SIAM J. Math. Analysis 11 (1980), 938-951.

3. W. N. Bailey, On the basic bilateral hypergeometric series ${ }_{2} \psi_{2}$, Quart. J. Math. Oxford 1 (1950), 194-198.

4. J. F. Van Diejen, On certain multiple Bailey, Rogers and Dougall type summation formulas, Publ. Res. Inst. Math. Sci. 33 (1997), 483-508.

5. J. F. Van Diejen and L. Vinet, The quantum dynamics of the compactified trigonometric Ruijsenaars-Schneider model, Comm. Math. Phys. 197 (1998), 33-74.

6. J. Dougall, On Vandermonde's theorem and some more general expansions, Proc. Edinburgh Math. Soc. 25 (1907), 114-132.

7. G. Gasper and M. Rahman, Basic Hypergeometric Series, Cambridge University Press, 1st edition, 1990.

8. R. A. Gustafson and C. Krattenthaler, Determinant evaluations and $U(n)$ extensions of Heine's ${ }_{2} \phi_{1}$ transformations. In: Special functions: $q$-series and related topics (M. Ismail, D. Masson, and M. Rahman, eds.), Fields Institute Communications, Vol. 14. Amer. Math. Soc., Providence, RI, 1997.

9. L. Habsieger, Une q-intégrale de Selberg-Askey, SIAM J. Math. Anal. 19 (1988), 1475-1489.

10. M. E. H. Ismail, $A$ simple proof of Ramanujan's ${ }_{1} \psi_{1}$ summation formula, Proc. Amer. Math. Soc. 63 (1977), 185-186. 
11. K. W. J. Kadell, A proof of Askey's conjectured q-analogue of Selberg's integral and a conjecture of Morris, SIAM J. Math. Anal. 19 (1988), 969-986.

12.,$A$ proof of the $q$-Macdonald-Morris conjecture for $B C_{n}$, Mem. Amer. Math. Soc. 516 (1994).

13. - The Selberg-Jack symmetric functions, Adv. in Math. 130 (1997), 33-102.

14. J. Kaneko, $A_{1} \Psi_{1}$ summation theorem for Macdonald polynomials, Ramanujan J., to appear.

15. _ Selberg integrals and hypergeometric functions associated with Jack polynomials, SIAM J. Math. Anal. 24 (1993), 1086-1110.

16. (1996), 1086-1110.

17. _ A triple product identity for Macdonald polynomials, J. Math. Anal. Appl. 200 (1996), 355-367.

18. — Constant term identities of Forrester-Zeilberger-Cooper, Discrete Math. 173 (1997), 79-90.

19. I. G. Macdonald, Hypergeometric functions, Unpublished manuscript.

20. _ Symmetric Functions and Hall Polynomials, Oxford University Press, Oxford, 2nd edition, 1995.

21. S. C. Milne, Balanced ${ }_{3} \phi_{2}$ summation theorems for $U(n)$ basic hypergeometric series, Adv. in Math. 131 (1997), 93-187.

22. E. M. Opdam, An analogue of the Gauss summation formula for hypergeometric functions related to root systems, Math. Z. 212 (1993), 313-336.

23. M. Shlosser, Summation theorems for multidimensional basic hypergeometric series by determinant evaluations, Univ. Vienna preprint, available from http://radon.mat.univie.ac.at/ People/mschloss, 1996.

24. Z. Yan, A class of generalized hypergeometric functions in several variables, Canad. J. Math. 44 (1992), 1317-1338.

25. D. Zeilberger, A Stembridge-Stanton style elementary proof of the Habsieger-Kadell q-Morris identity, Discrete. Math. 79 (1989), 313-322.

Department of Mathematics, University of Melbourne, Parkville, Victoria 3052, AusTRALIA 